\title{
A PROSPECTIVE STUDY TO CORRELATE ANGIOGRAPHIC PROFILE AND CLINICAL OUTCOME OF ACUTE CORONARY SYNDROME PATIENTS WITH ATRIAL FIBRILLATION
}

\author{
Sushant Kumar1, Yogesh Kothari², Rajiv Girdhar 3 , Swati Prasad ${ }^{4}$, Ram Anil Raj5, H. Venkataramaiah', Narayana Raju7, \\ Kumar Kenchappa ${ }^{8}$ \\ ${ }^{1}$ Senior Resident, Department of Cardiology, Rajarajeswari Medical College and Hospital, Mysore Road, Bangalore. \\ ${ }^{2}$ Associate Professor, Department of Cardiology, Rajarajeswari Medical College and Hospital, Mysore Road, Bangalore. \\ ${ }^{3}$ Senior Resident, Department of Cardiology, Rajarajeswari Medical College and Hospital, Mysore Road, Bangalore. \\ 4 Junior Resident, Department of Medicine, Rajarajeswari Medical College and Hospital, Mysore Road, Bangalore. \\ ${ }^{5}$ Assistant Professor, Department of Cardiology, Rajarajeswari Medical College and Hospital, Mysore Road, Bangalore. \\ ${ }^{6}$ Professor and HOD, Department of Cardiology, Rajarajeswari Medical College and Hospital, Mysore Road, Bangalore. \\ ${ }^{7}$ Assistant Professor, Department of Cardiology, Rajarajeswari Medical College and Hospital, Mysore Road, Bangalore. \\ ${ }^{8}$ Assistant Professor, Department of Cardiology, Rajarajeswari Medical College and Hospital, Mysore Road, Bangalore.
}

ABSTRACT

\section{BACKGROUND}

Despite advances in the diagnosis and management of Acute Coronary Syndrome (ACS), Atrial Fibrillation (AF) remains a commonly encountered complication leading to adverse short- and long-term outcomes across the whole spectrum of ACS. At present, the underlying mechanisms of AF in myocardial ischaemia remain incompletely understood.

This study evaluates the incidence and trends of new-onset AF in ACS, its impact on ACS management, the associated prognostic significance and its correlation with coronary artery disease.

\section{MATERIALS AND METHODS}

We analysed 100 consecutive patients with ACS between December 2014 and April 2016. Angiographic profile of patients with ACS with AF was compared. Cases were analysed with respect to Major Adverse Cardiac Events (MACE) and mortality during hospitalisation.

\section{RESULTS}

Out of 100 patients included in the study who developed AF, 96 of these had new-onset AF and 4 had previous AF. The AF group had a higher risk profile for MACE including those with hypertension history, prior infarct, hypotension on admission, tachycardia on presentation, a higher Killip class, non-ST elevation MIs and inferior infarcts. Moreover, patients with new-onset AF were more likely to have left main coronary artery disease versus patients with a prior AF history. In all 100 patients who underwent coronary angiogram, it revealed significant SVD in 40 cases, DVD in 32 cases, TVD in 20 cases, insignificant CAD in 4 cases and normal coronaries in 4 cases. The in-hospital mortality for new-onset AF, previous AF and non-AF patients were 14, 11.6 and 5.2\% respectively. New onset AF persisted as an independent predictor for mortality in patients with significant coronary artery disease (involvement of Left Main, LAD, LCX, RCA).

\section{CONCLUSION}

$\mathrm{AF}$ is not an infrequent event during ACS. The presence of new onset AF with significant coronary artery disease is associated with nearly a $60 \%$ increase in mortality with MI patients. AF patients had a poorer prognosis with a higher incidence of malignant arrhythmias, reinfarction, ischaemic mitral regurgitation, heart failure, cardiogenic shock and inhospital mortality than non-AF patients. Our results suggest that the appearance of NAF during ACS should not be considered an isolated benign event, but a severe complication with prognosis implications.

\section{KEYWORDS}

AF: Atrial Fibrillation; HF: Heart Failure; ACS: Acute Coronary Syndrome; CV: Cardiovascular; NAF- New Onset AF; Pr AF- Previous AF, SVD- Single Vessel Disease, DVD- Double Vessel Disease, TVD- Triple Vessel Disease, CAD- Coronary Artery Disease.

HOW TO CITE THIS ARTICLE: Kumar S, Kothari Y, Girdhar R, et al. A prospective study to correlate angiographic profile and clinical outcome of acute coronary syndrome patients with atrial fibrillation. J. Evolution Med. Dent. Sci. 2017;6(36):2977-2981, DOI: $10.14260 /$ Jemds/2017/641

Financial or Other, Competing Interest: None.

Submission 24-03-2017, Peer Review 22-04-2017,

Acceptance 27-04-2017, Published 04-05-2017.

Corresponding Author:

Dr. Sushant Kumar

Senior Resident,

Department of Cardiology,

Rajarajeswari Medical College and

Hospital, Mysore Road, Bangalore-560074.

E-mail: ssushant55@yahoo.com

DOI: $10.14260 /$ jemds $/ 2017 / 641$

\section{BACKGROUND}

Atrial Fibrillation (AF) is the most common cardiac arrhythmia observed in clinical practice and a frequent complication of Acute Coronary Syndromes (ACS) with an incidence ranging between 5 and 23\%.1-13 The prognostic implication of AF in the ACS setting is unclear. Some studies have found an association between AF and increased morbidity and mortality,6,7,9,14 whereas other studies have failed to detect this association.1,8 Majority of these findings were derived from studies using unadjusted multivariate models ${ }^{2-10}$ or post-hoc analyses of randomised controlled trials ${ }^{6-7,9,14}$; only few studies 
differentiated relation between AF with coronary artery disease.

This study aims to determine the angiographic profile and clinical outcome of ACS patients with atrial fibrillation and to identify risk factors for its development and to determine if AF during ACS is an independent risk factor for in-hospital mortality.

\section{MATERIALS AND METHODS \\ Data Source}

In brief, data from patients admitted to coronary care units in Raja Rajeswari Medical College and Hospital, Bangalore with the diagnosis of ACS were collected from December 2014 to April 2016. The study and this article followed the ethics and privacy guidelines of the independent local ethics and research committees.

\section{Study Group}

The study group includes patients with ACS who develop AF on 12-lead ECG during hospital stay or by history of AF or documented on Holter study.

\section{Variables}

ST-segment Elevation Myocardial Infarction (STEMI) was defined based on the following criteria: acute ischaemic symptoms and ST-segment elevation $\geq 2 \mathrm{mV}$ in two contiguous precordial leads as well as $\geq 1 \mathrm{mV}$ in inferior leads or a presumed/new left bundle branch block on initial electrocardiogram. Patients without persistent ( $>20$ mins) STsegment elevation in two or more contiguous leads, but with biomarker evidence of myocardial necrosis are classified as NSTEMI. Unstable angina was defined as the presence of symptoms of angina at rest, new-onset angina or accelerated ischaemic symptoms without electrocardiographic changes and elevation of cardiac enzymes. Cardiogenic shock was defined as having clinical signs of pulmonary congestion and impaired end-organ perfusion with persistent hypotension defined as a systolic blood pressure less than $90 \mathrm{mmHg}$ for more than 30 mins or the need for vasopressor therapy to maintain a systolic pressure above $90 \mathrm{mmHg}$.

\section{Statistical Analysis}

Data are expressed as the mean \pm standard deviation using SPSS software.

\section{Patient Characteristics}

Baseline demographic and socioeconomic characteristics including age, gender, race, region of residence, employment status, education status, marital status and living arrangements were examined.

\section{Clinical Data}

The baseline characteristics are shown in Table 1 . AF patients in totality were more likely to be male, older and sicker than normal sinus rhythm patients. The NAF patients presented with less comorbidity, but showed a worse clinical presentation at admission with a greater proportion of patients with STEMI including anterior MI, a higher Killip class and cardiac troponin levels and low LVEF with propensity for high ventricular arrhythmias.
RESULTS

\begin{tabular}{|c|c|c|c|}
\hline Characteristic & $\begin{array}{c}A F \\
(n=100)\end{array}$ & $\begin{array}{l}\text { Previous } \\
\text { AF }(n=4)\end{array}$ & $\begin{array}{l}\text { New AF } \\
(n=96)\end{array}$ \\
\hline $\begin{array}{l}\text { Female } \\
\text { Male }\end{array}$ & $\begin{array}{l}10 \\
90\end{array}$ & $\begin{array}{l}0 \\
4 \\
\end{array}$ & 96 \\
\hline Age (years) & $61.2 \pm 5$ & $61 \pm 10$ & $61 \pm 9.6$ \\
\hline Smoking & 80 & 2 & 78 \\
\hline $\mathrm{BMI} \geq 30(\mathrm{~kg} / \mathrm{m} 2)$ & 70 & 2 & 68 \\
\hline Diabetes & 20 & 2 & 18 \\
\hline Hypertension & 40 & 4 & 36 \\
\hline Previous MI & 8 & 0 & 8 \\
\hline Prior CHF & 2 & 2 & 0 \\
\hline AF History & - & 4 & - \\
\hline Peripheral Arterial Disease & 2 & & 2 \\
\hline $\begin{array}{c}\text { Renal Impairment } \\
(\mathrm{CrCl}<60 \mathrm{~mL} / \mathrm{min}) \\
\end{array}$ & 10 & 0 & 10 \\
\hline Previous PCI & 2 & 2 & 0 \\
\hline Prior CV Surgery & 4 & 2 & 2 \\
\hline $\mathrm{VF}$ & 6 & 0 & 6 \\
\hline Systolic BP (mmHg) 110 & 80 & - & 80 \\
\hline Heart Rate $(\mathrm{bpm})$ & $96 \pm 24$ & $91 \pm 29$ & $95 \pm 20$ \\
\hline LVEF $(<40 \%)$ & 80 & 4 & 76 \\
\hline $\begin{array}{l}\text { Cardiac troponin/ } \\
\text { CPKMB High Value }\end{array}$ & 100 & 0 & 100 \\
\hline \multicolumn{4}{|l|}{ Killip class } \\
\hline $\mathrm{I}$ & 80 & - & 80 \\
\hline II & & - & \\
\hline III & & - & - \\
\hline IV & 20 & - & 20 \\
\hline
\end{tabular}

\begin{tabular}{|c|c|}
\hline Cases & Number (Percentage) \\
\hline STEMI & $76(76 \%)$ \\
\hline NSTEMI & $4(4 \%)$ \\
\hline Unstable Angina & $20(20 \%)$ \\
\multicolumn{2}{|c|}{ Table 2. Distribution of STEMI, } \\
NSTEMI, USA in Study Population \\
\hline
\end{tabular}

This study included 100 patients, who developed AF during hospitalisation. Of these, 96 (96\%) patients developed NAF and $4(4 \%)$ had previous AF. Of all these $76(76 \%)$ patients had STEMI, 4 (4\%) patients had NSTEMI and $20(20 \%)$ unstable angina.

Among STEMI 52 (52\%) patients had AWMI, 4 (4\%) patients had AWMI with re-infarction, $6(6 \%)$ patients of AWMI developed VT which reverted to normal sinus rhythm with DC cardioversion; 18 (18\%) patients had combined IWMI + PWMI and $4(4 \%)$ patients had combined IWMI + PWMI + RVMI. Among ACS with unstable angina patients, 2 (2\%) had RHD S/P AVR, S/P PTCA STENT TO LMCA $\rightarrow$ LAD with stent patent. Other $4(4 \%)$ patients had RHD moderate MR, 2 (2\%) patients had HOCM with unstable angina. Another patient had HCM with unstable angina; 4 (4\%) patients were post-CABG with Graft to LIMA $\rightarrow$ LAD and SVG $\rightarrow$ OM.

\begin{tabular}{|c|c|}
\hline Cases & Number (Percentage) \\
\hline AWMI & $52(52 \%)$ \\
\hline IWMI + PWMI & $18(18 \%)$ \\
\hline IWMI + RVMI + LWMI & $4(4 \%)$ \\
\hline ACS Unstable Angina with RHD MR & $4(4 \%)$ \\
\hline ACS Unstable Angina S/P CABG S & $4(4 \%)$ \\
\hline $\begin{array}{c}\text { ACS Unstable Angina S/P PTCA } \rightarrow \\
\text { LMCA } \rightarrow \text { LAD, S/P AVR }\end{array}$ & $2(2 \%)$ \\
\hline $\begin{array}{r}\text { Table 3. STEMI, NSTEMI, USA } \\
\text { Distribution in Study Population }\end{array}$ \\
\hline
\end{tabular}




\begin{tabular}{|c|c|}
\hline Sex & Number (Percentage) \\
\hline Male & $90(90 \%)$ \\
\hline Female & $10(10 \%)$ \\
\hline \multicolumn{2}{|c|}{ Table 4. Gender Distribution in Study Population } \\
\hline
\end{tabular}

\begin{tabular}{|c|c|}
\hline Age/Comorbidity & Number (Percentage) \\
\hline Age $>50$ Yrs. & $84(84 \%)$ \\
\hline Age $<50$ Yrs. & $16(16 \%)$ \\
\hline HTN & $40(40 \%)$ \\
\hline T2DM & $20(20 \%)$ \\
\hline \multicolumn{2}{|c|}{ Table 5. Distribution of HTN, T2DM in Study Population } \\
\hline
\end{tabular}

$90(90 \%)$ patients were male and $10(10 \%)$ patients were females; 84 (84\%) patients were aged above 50 years and 16 (16\%) patients were aged below 50 years; 40 (40\%) patients had hypertension and 20 (20\%) patients had associated diabetes. Echocardiogram done in all patients revealed LV Dysfunction with LVEF below 40\% in 80 (80\%) patients. Rest of the patients had normal LVEF.

\begin{tabular}{|c|c|}
\hline Coronary Artery Disease & Number (Percentage) \\
\hline SVD & $40(40 \%)$ \\
\hline DVD & $32(32 \%)$ \\
\hline TVD & $20(20 \%)$ \\
\hline Normal Coronaries & $4(4 \%)$ \\
\hline Insignificant CAD & $4(4 \%)$ \\
\hline \multicolumn{2}{|c|}{ Table 6. Distribution of CAD in } \\
Study Population in Percentage \\
\hline
\end{tabular}

\begin{tabular}{|c|c|}
\hline Coronary Artery Disease & Number of Cases (\%) \\
\hline LAD & $72(72 \%)$ \\
\hline LCX & $42(42 \%)$ \\
\hline RCA & $46(46 \%)$ \\
\hline Table 7. Distribution of CAD in \\
Study Population in Percentage \\
\hline
\end{tabular}

Coronary Angiogram done in all 100 cases, which revealed SVD (Single Vessel Disease) in $40(40 \%)$ cases, DVD (Double Vessel Disease) in 32 (32\%) cases, TVD (Triple Vessel Disease) in $20(20 \%)$ cases, $4(4 \%)$ cases had insignificant coronary artery disease and in $4(4 \%)$ cases coronary angiogram was normal. LAD lesion was found in $72(72 \%)$ cases with average lesion of $>70 \%$ stenosis. LCX has significant lesion in $42(42 \%)$ cases with average $80 \%$ stenosis. RCA has significant lesion in $46(46 \%)$ cases with average stenosis of $80 \%$.

\begin{tabular}{|c|c|}
\hline Coronary Artery & Number of Cases (Percentage) \\
\hline Prox LAD & $48(48 \%)$ \\
\hline Mid LAD & $22(22 \%)$ \\
\hline Distal LAD & $4(4 \%)$ \\
\hline \multicolumn{2}{|c|}{ Table 8. Distribution of LAD Lesion } \\
in Study Population in Percentage
\end{tabular}

\begin{tabular}{|c|c|}
\hline Coronary Artery Disease & Number (Percentage) \\
\hline Prox LCX & $24(24 \%)$ \\
\hline Distal LCX & $6(6 \%)$ \\
\hline
\end{tabular}

Significant proximal LAD lesion was found in 48 (48\%) cases with $14(14 \%)$ cases had total occlusion in proximal segment and 4 (4\%) cases had subtotal occlusion; 22 (22\%) cases had Mid LAD lesion with average stenosis of 70\%; 4 (4\%) cases had combined lesion in proximal and mid segment of
LAD with stenosis of $90 \%$ and $70 \%$ respectively; 12 (12\%) cases had LAD lesion, which was insignificantly affected. Proximal segment of LCX was affected in 24 (24\%) cases with average stenosis of $80 \%$. Distal segment of LCX had significant lesion in $6(6 \%)$ cases. Major OM had significant lesion in proximal segment in $4(4 \%)$ cases with average stenosis of $80 \%$. LCX has no flow limiting lesion in $4(4 \%)$ cases. LCX was Non-dominant vessel in $96(96 \%)$ cases and in $4(4 \%)$ cases co-dominant vessel. Ramus has significant lesion in 4 (4\%) cases.

\begin{tabular}{|c|c|}
\hline Coronary Artery Disease & Number (Percentage) \\
\hline Prox RCA & $22(22 \%)$ \\
\hline Mid RCA & $26(26 \%)$ \\
\hline Distal RCA & $6(6 \%)$ \\
\hline
\end{tabular}

\begin{tabular}{|c|c|}
\hline Parameters & STEMI with AF \\
\hline LVEF < 40\% & $80(80 \%)$ \\
\hline Killip CLASS IV & $20(20 \%)$ \\
\hline Arrhythmias & $6(6 \%)$ \\
\hline Reinfarction & $4(4 \%)$ \\
\hline \multicolumn{2}{|c|}{ Table 11. Prognostic Markers in STEMI with AF } \\
\hline
\end{tabular}

RCA was found to be a dominant vessel in 96 (96\%) cases and was a co-dominant vessel in 4 (4\%) cases. RCA has significant lesion in $46(46 \%)$ cases with average stenosis of $80 \%$. Proximal segment of RCA has significant lesion in 22 (22\%) cases. Mid segment has significant lesion in 26 (26\%) cases and distal segment of RCA is affected in $6(6 \%)$ cases. Proximal segment of RCA has total occlusion in 12 (12\%) cases; $6(6 \%)$ cases of RCA has diffuse disease and 8 cases has insignificant disease.

AF group had worse Killip class at admission, higher troponin level and the Left Ventricle Ejection Fraction (LVEF) was lower in AF patients than in non-AF patients.

\section{DISCUSSION}

Atrial Fibrillation (AF) is characterised by irregular, disorganised and chaotic electrical activity of the atrium. It is a common arrhythmia and major cause of morbidity and mortality not only in the Western world, but in India as well. AF complicates approximately $10 \%-18 \%$ of acute infarcts. We have demonstrated an increased risk of mortality associated with the presence of AF in the setting of ACS. NAF is associated with an almost $60 \%$ increase in mortality risk. This increased risk was not demonstrated for previous AF after a two wellconducted adjustment analyses. The results of previous studies are conflicting regarding the prognostic impact of $\mathrm{AF}$ types in MI.1-6,8,9 However, our results are consistent with some previous reports. ${ }^{2-13}$

The predictors and significance of AF in the setting of MI reviewed by Schmitt et al ${ }^{11}$ are fairly consistent with our findings. Schmitt et al concluded that AF during MI with significant coronary artery disease implied worse short- and long-term outcomes, independent of MI treatment strategies. Two well-conducted meta-analyses focusing on the prognostic implication of AF during MI have been published. Jabre et $\mathrm{al}^{12}$ included 43 articles with a total of 278,854 MI patients indicated that AF led to a $40 \%$ increase in the risk of mortality in the setting of MI, even after adjustment for the same variables as in our study and concluded that NAF and Previous AF did not differ in mortality risk. 
The second and most recent study by Angeli et al ${ }^{13}$ was a meta-analysis of 24 studies comparing outcomes in patients with MI and sinus rhythm with those observed for AF patients. In their study, patients with AF with significant coronary artery disease (involvement of LAD, LCX and RCA) had a 2-fold increased risk of in-hospital mortality and patients with clear evidence of NAF had a 3-fold higher risk of mortality than sinus rhythm patients, although the study did not adjust for the Killip class, an important confounding variable. ${ }^{15,16}$

It is important to note that in the meta-analysis by Jabre et $\mathrm{al}^{12}$ the association between AF, coronary artery disease and high mortality was similar for NAF and Previous AF. These results persisted even after adjusting for confounding variables such as heart failure and revascularisation, although only four of the 43 studies included in his work performed such important adjustments. These results are consistent with ours with respect to risk ratios for NAF patients.

NAF in the setting of ACS reflects a greater ischaemic burden than Previous AF as shown by the lower LVEF, larger infarct sizes and higher Killip class associated with NAF compared with Previous AF in our study. In contrast, Previous $\mathrm{AF}$ is associated with a worse clinical baseline profile than NAF due to the existence of chronic heart disease. ${ }^{9}$ Along the same line of reasoning, recent data from a large registry in Canada ${ }^{17}$ has showed that patient with prior history of AF receive less evidence-based therapies and in-hospital coronary angiography compared to non-AF patients, similarly to our results. As in our observational study, patients with prior $\mathrm{AF}$ were older and sicker ${ }^{17}$ and broadly differ to those included in the work by Lopes et $\mathrm{al}^{18}$ that examined pooled data from 10 ACS trials, which includes more cases of NAF and STEMI and probably less patients with significant comorbidities or those receiving warfarin, as is common in patients included in clinical trials compared with those derived from registries.

The development of AF may worsen the prognosis of ACS due to several factors. First, AF triggers some adverse haemodynamic effects including increased oxygen consumption, loss of atrial contraction and AV synchrony, which lead to a decrease in effective cardiac output.7,9,12-15,19 Secondly, irregular RR intervals can develop malignant arrhythmias as was observed in our cohort. ${ }^{12-15}$ However, the mechanism of AF-related mortality in ACS remains elusive; the association of $\mathrm{AF}$ with higher heart rates, lower LVEF and an increased incidence of HF suggests that haemodynamic impairment is a potential explanation as well as increased adrenergic and neurohormonal activation. ${ }^{9,12,13}$

The rate of cerebrovascular events in patients with any type of AF in our study was quite low and not significantly different from patients in sinus rhythm. This may have been due to the relatively young age of patients enrolled in our ACS registry, but we cannot rule out other reasons.

\section{Study Limitations}

Several limitations of the present investigation deserve discussion. First, the study population and data concerning the temporal relation between the onset of $\mathrm{AF}$ and the appearance of complications were not available, so we can establish an association but not a causality relationship. Silent episodes of AF could not be analysed in the sinus rhythm group. Furthermore, the adjustment of variables that were not measured at baseline (e.g. mechanical complications of MI and revascularisation) in multivariable analysis and comparison of outcomes which might occur before or after AF (since its timing was not known) should be interpreted with caution. Secondly, data concerning the causes of death were only available for a minority of patients; thus, the causes of death are not shown. Data concerning treatment strategies used for $\mathrm{AF}$ as well as the timing, duration and final outcome of $\mathrm{AF}$ episodes were not assessed.

\section{CONCLUSION}

$\mathrm{AF}$ is not an infrequent event during ACS. The presence of NAF (New onset AF) with significant coronary artery disease (Involvement of LAD, LCX and RCA) is associated with nearly a $60 \%$ mortality increase in MI patients. AF patients had a poorer prognosis with a higher incidence of malignant arrhythmias, reinfarction, ischaemic mitral regurgitation, heart failure, cardiogenic shock and in-hospital mortality than non-AF patients. Also, it is likely that patients who are older and sicker such as those with atrial fibrillation with severe LV dysfunction had more extensive coronary artery disease. Our results suggest that the appearance of NAF during ACS should not be considered an isolated benign event, but a severe complication with prognosis implications. These results are hypothesis generating and the prognostic impact of treatment strategies for NAF in MI patients must be assessed in future studies.

\section{REFERENCES}

[1] Goldberg RJ, Seeley D, Becker RC, et al. Impact of atrial fibrillation on the in-hospital and long-term survival of patients with acute myocardial infarction: a community-wide perspective. Am Heart J 1990;119(5):996-1001.

[2] Sakata K, Kurihara H, Iwamori K, et al. Clinical and prognostic significance of atrial fibrillation in acute myocardial infarction. Am J Cardiol 1997;80(12):15227.

[3] Torres M, Rocha S, Marques J, et al. Impact of atrial fibrillation in acute coronary syndromes. Rev Port Cardiol 2008;27(11):1407-18.

[4] Cui K, Gu S, Ding YS, et al. Effects of atrial fibrillation/atrial flutter on the short and medium-term prognosis of patients with acute myocardial infarction. J Interv Radiol 2008;17:594-6.

[5] Galcera TJ, Melgarejo MA, Garcia AA, et al. Incidence, clinical characteristics and prognostic significance of supraventricular tachyarrhythmias in acute myocardial infarction. Rev Esp Cardiol 1999;52(9):647-55.

[6] Lopes RD, Elliott LE, White HD, et al. Antithrombotic therapy and outcomes of patients with atrial fibrillation following primary percutaneous coronary intervention: results from the APEX-AMI trial. Eur Heart J 2009;30(16):2019-28.

[7] Crenshaw BS, Ward SR, Granger CB, et al. Atrial fibrillation in the setting of acute myocardial infarction: the GUSTO-I experience. Global utilization of streptokinase and TPA for occluded coronary arteries. J Am Coll Cardiol 1997;30(2):406-13. 
[8] Kinjo K, Sato H, Sato H, et al. Prognostic significance of atrial fibrillation/atrial flutter in patients with acute myocardial infarction treated with percutaneous coronary intervention. Am J Cardiol 2003;92(10):1150-4.

[9] Pizzetti F, Turazza FM, Franzosi MG, et al. Incidence and prognostic significance of atrial fibrillation in acute myocardial infarction: the GISSI-3 data. Heart 2001;86(5):527-32.

[10] Rathore SS, Berger AK, Weinfurt KP, et al. Acute myocardial infarction complicated by atrial fibrillation in the elderly: prevalence and outcomes. Circulation 2000;101(9):969-74.

[11] Schmitt J, Duray G, Gersch BJ, et al. Atrial fibrillation in acute myocardial infarction: a systematic review of the incidence, clinical features and prognostic implications. Eur Heart J 2009;30(9):1038-45.

[12] Jabre P, Roger VL, Murad MH, et al. Mortality associated with atrial fibrillation in patients with myocardial infarction: a systematic review and meta-analysis. Circulation 2011;123(15):1587-93.

[13] Angeli F, Reboldi G, Garofoli M, et al. Atrial fibrillation and mortality in patients with acute myocardial infarction: a systematic overview and meta-analysis. Curr Cardiol Rep 2012;14(5):601-10.
[14] Behar S, Zahavi Z, Goldbourt U, et al. Long-term prognosis of patients with paroxysmal atrial fibrillation complicating acute myocardial infarction. SPRINT study group. Eur Heart J 1992;13(1):45-50.

[15] Liberthson RR, Salisbury KW, Hutter AM, et al. Atrial tachyarrhythmias in acute myocardial infarction. Am J Med 1976;60(7):956-60.

[16] Wang TJ, Larson MG, Levy D, et al. Temporal relations of atrial fibrillation and congestive heart failure and their joint influence on mortality: the Framingham heart study. Circulation 2003;107(23):2920-5.

[17] Al Khdair D, Alshengeiti L, Elbarouni B, et al. Management and outcome of acute coronary syndrome patients in relation to prior history of atrial fibrillation. Can J Cardiol 2012;28(4):443-9.

[18] Lopes RD, Pieper KS, Horton JR, et al. Short- and longterm outcomes following atrial fibrillation in patients with acute coronary syndromes with or without STsegment elevation. Heart 2008;94(7):867-73.

[19] Waldecker B. Atrial fibrillation in myocardial infarction complicated by heart failure: cause or consequence? Eur Heart J 1999;20(10):710-2. 